Arq. Bras. Med. Vet. Zootec., v.68, n.1, p.127-135, 2016

\title{
Caracterização morfológica do esôfago de tartarugas-verdes (Chelonia mydas)
}

\author{
[Morphologic characterization of the esophagus of green turtles (Chelonia mydas)] \\ A. Calais Júnior, J.A.O. David, L.C. Nunes* \\ Universidade Federal do Espírito Santo - Alegre, ES
}

\begin{abstract}
RESUMO
A tartaruga-verde, Chelonia mydas (Linnaeus, 1758), é a mais comum na região costeira do Brasil e a compreensão da morfologia e de processos fisiológicos básicos nesses organismos é importante, podendo fornecer subsídios para estudos evolutivos sobre a espécie. O objetivo deste trabalho foi descrever a morfologia do esôfago de C. mydas, com a finalidade de se produzirem informações que possibilitem análises comparativas com outros quelônios. Utilizaram-se oito animais, provenientes do litoral do Espírito Santo, no período de setembro a novembro de 2014. Obtiveram-se dados de comprimento curvilíneo da carapaça (CCC) e largura curvilínea da carapaça (LCC). O esôfago foi retirado desde a inserção da orofaringe até a porção inicial do estômago para avaliação histomorfológica e medida do comprimento. Foram avaliadas quatro regiões do esôfago. O órgão inteiro foi fixado em formalina a $10 \%$ e, posteriormente, foi coletado um fragmento de cada região para processamento histológico e coloração pelas técnicas hematoxilina e eosina (HE), tricrômico de Masson, Gram e ácido periódico-Schiff (PAS). Os animais juvenis possuíam CCC médio de $38,8 \pm 6,43 \mathrm{~cm}$ e LCC média de $35,6 \pm 6,59 \mathrm{~cm}$; o animal adulto mediu $102,3 \mathrm{~cm}$ de CCC e $96,9 \mathrm{~cm}$ de LCC. O comprimento médio do esôfago foi de $20,47 \pm 1,56$ nos juvenis, e total de $55,6 \mathrm{~cm}$ no adulto. Observou-se que o esôfago de C. mydas é um órgão tubular muscular constituído de papilas cônicas que variam de quantidade e tamanho à medida que se aproximam do estômago, revestidas por epitélio estratificado pavimentoso queratinizado e ricas em tecido mixoide. Há variação nas camadas mucosa, muscular externa e serosa entre as regiões do esôfago e há presença de glândulas produtoras de muco na mucosa da região da junção gastroesofágica. As características histomorfológicas do esôfago de C. mydas são importantes para exercer as funções mecânica e de proteção da mucosa desse órgão.
\end{abstract}

Palavras-chave: morfologia, quelônios, trato gastrintestinal, papilas esofágicas

\begin{abstract}
Understanding the morphology and basic physiological processes in Chelonia mydas is extremely important and can provide support for evolutionary studies on the species. The aim of this study was to describe the morphology of $\mathrm{C}$. mydas' esophagus in order to produce data that allows comparative analyses with other turtles. Eight specimens of C. mydas from the coast of Espírito Santo were used during the period from September to November 2014. We obtained the curved carapace length (CCL) and curved carapace width (CCW) in centimetres. The esophagus was completely removed from the insertion region of the oropharynx to the initial portion of the stomach for macroscopic and microscopic evaluation. The measurement on the length and description of the structures in four regions of the esophagus were performed. The entire organ was fixed in 10\% formalin and then a one centimetre diameter fragment was collected for histological processing and staining by hematoxylin and eosin (HE), Masson, Gram and periodic acid-Schiff (PAS) techniques. Juvenile animals had average CCL of $38.8 \pm$ $6.43 \mathrm{~cm}$ and average CCW of $35.6 \pm 6.59 \mathrm{~cm}$; the adult animal had a CCL measurement of $102.3 \mathrm{~cm}$ and CCW of $96.9 \mathrm{~cm}$. The average length of the esophagus was $20.47 \pm 1.56$ in youth, and total $55.6 \mathrm{~cm}$ in the adult. It was observed that $\mathrm{C}$. mydas' esophagus is a tubular muscular organ that consists of conical
\end{abstract}

Recebido em 25 de junho de 2015

Aceito em 27 de novembro de 2015

* Autor para correspondência (corresponding author)

E-mail: louisiane.nunes@ufes.br 
papillae that vary in quantity and size as they approach the stomach, lined by stratified squamous keratinized epithelium and rich in myxoid tissue. There is variation in the mucosal, submucosal, external muscle and serous layers among the four regions of the esophagus and there are mucus-producing glands in the submucosa of the gastroesophageal junction region. The histomorphometric characteristics of $\mathrm{C}$. mydas' esophagus are important to engage the mechanical function and mucosal protection of this organ.

Keywords: chelonian, morphology, gastrointestinal tract, esophageal papillae

\section{INTRODUÇÃO}

Vítimas de capturas acidentais, as tartarugas apresentam taxas consideráveis de mortalidade de espécies não alvo (Lewison et al., 2004). Dessa forma, observa-se um declínio global nas populações, em que seis das sete espécies existentes são categorizadas como vulneráveis, em perigo ou criticamente ameaçadas na Lista Vermelha da União Internacional para a Conservação da Natureza e dos Recursos Naturais - IUCN (IUCN, 2014).

Segundo Wallace et al. (2010), capturas por pesca acidental são as maiores ameaças para a conservação desses indivíduos, com relatos de aproximadamente 85000 animais capturados incidentalmente em todo o mundo, no período de 1990 a 2008. Entretanto, essas estimativas são limitadas devido à baixa confiabilidade das informações globais e à inexistência de relatos sobre as capturas em pescas de pequena escala (Wallace et al., 2010; Casale, 2011), associadas à falta de dados sobre a taxa de sobrevivência de animais liberados (Mangel et al., 2011).

Estudos morfológicos sobre o trato digestivo das tartarugas marinhas podem produzir informações que permitirão análises comparativas com outros quelônios, bem como fornecer subsídios para futuros estudos evolutivos sobre o grupo, especialmente as que dizem respeito a adaptações relacionadas com os hábitos alimentares (Magalhães et al., 2012).

Sabe-se que, em quelônios, o trato gastrointestinal é anatomicamente diversificado e a grande variedade de répteis exige mais estudos para compreensão de particularidades anatômicas (Costa et al., 2009). O esôfago é um tubo muscular cuja função é transportar o alimento da boca ao estômago e, de um modo geral, contém as mesmas camadas que o restante do trato digestório (Junqueira e Carneiro, 2013), entretanto, em tartarugas marinhas, apresenta papilas fortemente queratinizadas, que protegem a mucosa da dieta abrasiva e também podem atuar como dispositivos de filtragem (Elliott, 2007).

Portanto, o conhecimento morfológico de estruturas envolvidas em processos fisiológicos básicos nos organismos é extremamente importante na aquisição de conhecimentos ecológicos e evolutivos sobre as espécies. Por essa razão, objetivou-se com este trabalho descrever a morfologia do esôfago de Chelonia mydas, com a finalidade de produzir informações que possibilitem análises comparativas com outros quelônios.

\section{MATERIAL E MÉTODOS}

Foram utilizados oito espécimes de C. mydas (sete juvenis e um adulto), provenientes do litoral do Espírito Santo, encaminhados à base veterinária da empresa CTA Meio Ambiente, localizada no município de Anchieta, ES.

Todo protocolo experimental adotado possui autorização número 39329-2, do Sistema de Autorização e Informação em Biodiversidade (SisBio), vinculado ao Instituto Chico Mendes de Conservação da Biodiversidade (ICMBio) do Ministério do Meio Ambiente (MMA).

Com o intuito de minimizar a avaliação de animais com alterações cadavéricas significativas, coletou-se material apenas dos cadáveres que morreram durante o transporte do local de encalhe até a base ou dos animais que foram recebidos vivos na base para tratamento e vieram a óbito e não apresentaram lesão esofágica ao exame macroscópico, de setembro a novembro de 2014.

Os cadáveres foram submetidos à avaliação biométrica utilizando-se fita métrica. Obtiveram-se os dados de comprimento curvilíneo da carapaça (CCC) e de largura curvilínea da carapaça (LCC) em centímetro. Em seguida, foram necropsiados no Setor de 
Necroscopia da empresa CTA, seguindo a técnica de Wyneken (2001). O esôfago foi retirado completo, desde a região de inserção da orofaringe até a porção inicial do estômago, para avaliação macro e microscópica.

Para a avaliação macroscópica, o esôfago foi aberto na porção dorsal e obteve-se o comprimento total do órgão. Também foram avaliadas a presença, a distribuição e o formato das papilas esofágicas, bem como do esfíncter gastroesofágico. Foi feita a descrição morfológica das estruturas observadas, de acordo com a divisão de Junqueira e Carneiro (2013), sendo definidas quatro regiões, a saber: 1) junção gastroesofágica; 2) porção distal do esôfago; 3) porção medial e 4) porção proximal. Todo material foi fotodocumentado e fixado em formalina a $10 \%$.

O material foi encaminhado ao Laboratório de Patologia Animal da Universidade Federal do Espírito Santo e identificado com a letra "P", referente a material de pesquisa, seguido da sequência numérica de registro do setor e do ano. De cada porção esofágica foi coletado um fragmento de $1 \mathrm{~cm} \times 1 \mathrm{~cm}$ para processamento histológico de rotina para obtenção de cortes histológicos de $3 \mu \mathrm{m}$ de espessura em micrótomo manual e posterior coloração pelas técnicas hematoxilina e eosina (HE), tricrômico de Masson, Gram e ácido periódico-Schiff (PAS).

A avaliação microscópica considerou quatro camadas do tubo digestório, a saber: mucosa, submucosa, muscular externa e serosa ou adventícia. Todas as lâminas foram observadas e fotomicrografadas, respectivamente, com câmera digital Sony SteaddyShot DSC-W610, acoplada em microscópio óptico Bel A17.1026.

A análise estatística foi feita pelo método descritivo, com os dados expressos em porcentagem.

\section{RESULTADOS}

Entre os animais avaliados observou-se que os juvenis possuíam CCC médio de $38,8 \pm 6,43 \mathrm{~cm}$ e LCC média de $35,6 \pm 6,59 \mathrm{~cm}$, enquanto o animal adulto mediu $102,3 \mathrm{~cm}$ de CCC e $96,9 \mathrm{~cm}$ de LCC. Observou-se que o comprimento médio do

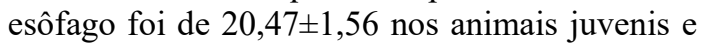
o total de 55,6cm no animal adulto (Tab. 1).

Tabela 1. Valores absolutos do comprimento curvilíneo da carapaça (CCC), da largura curvilínea da carapaça (LCC) e do comprimento total do esôfago de C. mydas do litoral do Espírito Santo, 2014

\begin{tabular}{cccc}
\hline Número da amostra & CCC $(\mathrm{cm})$ & LCC $(\mathrm{cm})$ & Comprimento total do esôfago $(\mathrm{cm})$ \\
\hline P95/14 & 47,6 & 43,5 & 22,2 \\
P97/14 & 42,2 & 36,6 & 21,9 \\
P118/14 & 36,5 & 36,1 & 20,6 \\
P123/14 & 39,6 & 36,9 & 21,3 \\
P124/14 & 33,9 & 32,4 & 18,8 \\
P125/14 & 43,6 & 41,0 & 20,5 \\
P128/14 & 102,3 & 96,9 & 55,6 \\
P129/14 & 28,5 & 23,1 & 18,0 \\
\hline
\end{tabular}

CCC: comprimento curvilíneo da carapaça; LCC: largura curvilínea da carapaça.

Em relação à anatomia do esôfago de C. mydas, verificou-se, em todos os casos, que se trata de um órgão tubular muscular localizado medialmente na região cervical, com desvio lateral para a esquerda na cavidade celomática, próximo ao coração.
Observou-se que, nas porções proximal, medial e distal, a mucosa esofágica é caracterizada por papilas em formato de cone, pontiagudas, orientadas caudalmente para o estômago. Notouse que essas papilas são delgadas e numerosas na porção proximal e aumentam de comprimento e diâmetro à medida que chegam à região medial, entretanto, há variação quanto à sua distribuição 


\section{Calais Júnior et al.}

e quantidade na região distal do esôfago, tornando-se menos numerosas. $\mathrm{Na}$ junção gastroesofágica, observou-se ausência das papilas cônicas pontiagudas, presença de pregas reticulares longitudinais e um estreitamento da musculatura caracterizando o esfíncter gástrico (Fig. 1).

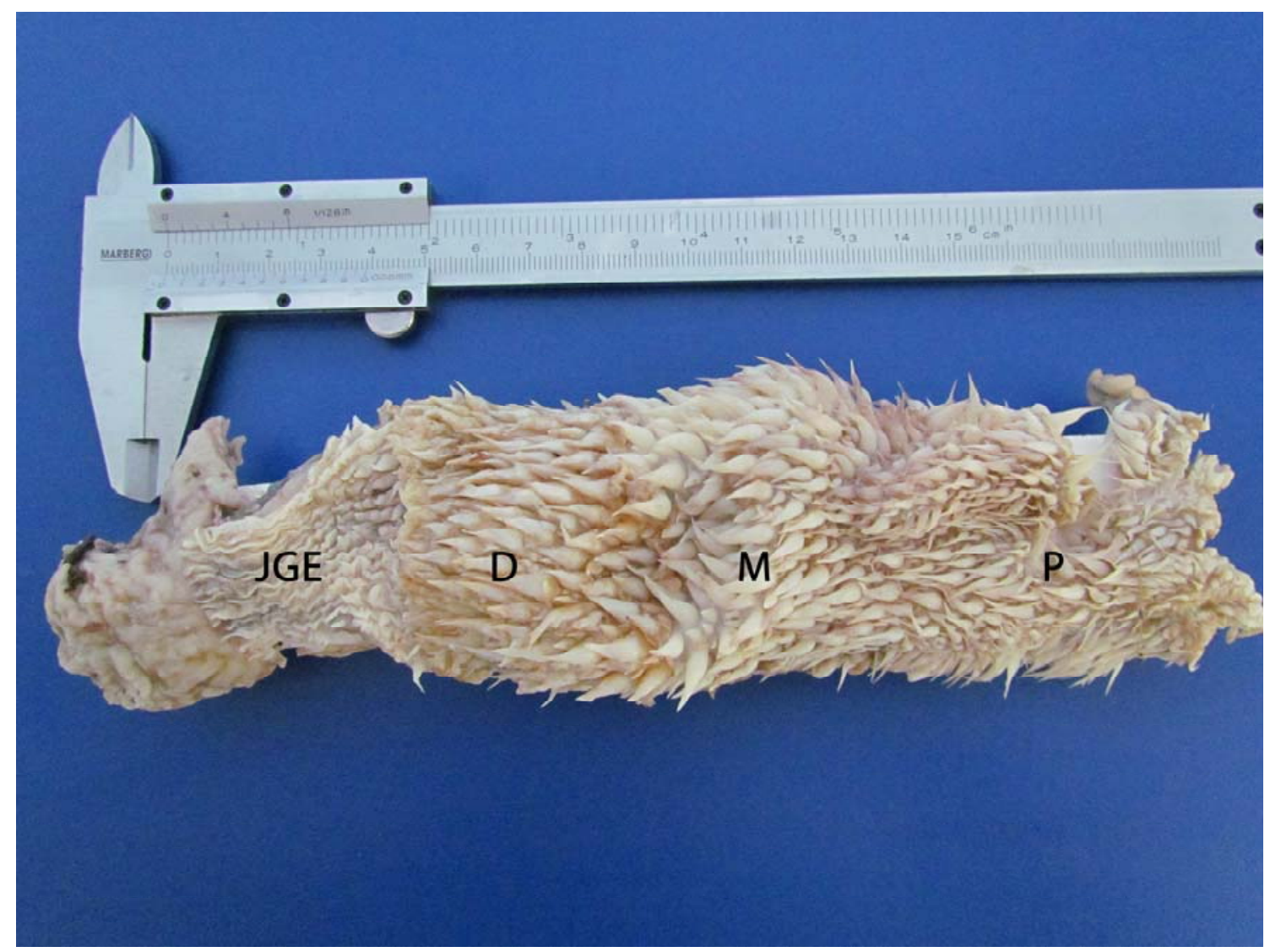

Figura 1. Fotomacrografia do esôfago normal de C. mydas proveniente do litoral do Espírito Santo evidenciando mucosa constituída de papilas cônicas, pontiagudas, orientadas caudalmente para o estômago, sendo as papilas delgadas na região proximal $(\mathrm{P})$, aumentadas de diâmetro e altura na região medial (M) e de número reduzido na região distal (D). Estreitamento da musculatura caracterizando o esfíncter gástrico (JGE).

Histologicamente, as quatro regiões do esôfago avaliadas neste estudo apresentaram três camadas distintas: mucosa, muscular externa e serosa. Não se observaram muscular da mucosa e submucosa distintas.

Na junção gastroesofágica, observou-se mucosa pregueada constituída por epitélio estratificado pavimentoso queratinizado e lâmina própria de tecido conjuntivo frouxo contendo vasos sanguíneos, nódulos linfoides e glândulas produtoras de muco, positivas ao PAS (Fig. 2B). A camada muscular externa é composta por músculo estriado esquelético, sendo a camada mais interna de disposição longitudinal e a mais externa circular. A camada de musculatura longitudinal é espessa, e a circular delgada nessa porção. A serosa é composta por tecido conjuntivo frouxo, revestida por mesotélio.

A porção distal revelou mucosa constituída por papilas cônicas pontiagudas em quantidade moderada, revestidas por epitélio estratificado pavimentoso queratinizado. A lâmina própria é semelhante à porção da junção gastroesofágica, mas não possui glândulas mucosas (Fig. 2C). A camada muscular externa é composta por musculatura estriada esquelética, sendo a camada mais interna de disposição longitudinal e a 
externa circular com distribuição semelhante à região anterior.

As porções proximal e medial revelaram mucosa constituída por papilas cônicas pontiagudas, revestidas por epitélio estratificado pavimentoso queratinizado. Na lâmina própria, há abundante tecido conjuntivo mixoide sustentando as papilas. Também não se verificaram estruturas glandulares. A camada muscular externa é composta por músculo estriado esquelético, sendo a camada mais interna de disposição circular e a mais externa longitudinal, diferindose das demais porções. A serosa é composta por tecido conjuntivo denso. Observou-se, ainda, que, na porção proximal, as papilas cônicas pontiagudas cornificadas apresentaram-se mais delgadas e mais baixas em relação às da região medial e que, no início da porção proximal, há adventícia em vez de serosa (Fig. 2A, 2C e 2D).

Em relação à disposição das camadas musculares, observou-se uma variação entre as regiões do esôfago. Sabe-se que a camada muscular circular tem por função auxiliar na constrição do órgão, enquanto a longitudinal auxilia na dilatação do lúmen. Dessa forma, acredita-se que, nas regiões proximal e medial, haja maior capacidade constritora e, na região distal, maior dilatação para propulsão do alimento.



Fig. 2. Fotomicrografia do esôfago normal de C. mydas proveniente do litoral do Espírito Santo, evidenciando em A) mucosa constituída de papilas cônicas revestidas por epitélio estratificado pavimentoso queratinizado (seta) e compostas por tecido mixoide, camada muscular circular interna (círculo) na porção proximal, Gram (Barra $=200 \mu \mathrm{m})$; B) lâmina própria glandular na junção gastroesofágica com glândulas mucosas (seta) positivas ao PAS (Barra=200 $\mu \mathrm{m}$ ); C) mucosa constituída de papilas cônicas contendo abundante tecido mixoide (seta) na região proximal, HE (Barra=200 $\mu \mathrm{m})$; D) evidenciação do tecido mixoide da imagem anterior, HE (Barra=100 $\mu \mathrm{m})$. 


\section{DISCUSSÃO}

O comprimento médio do esôfago de C. mydas citado por Magalhães et al. $(2010 ; 2012)$ foi de $19,32 \pm 4,09 \mathrm{~cm}$. Esses dados são semelhantes aos valores de comprimento médio de esôfago obtidos neste estudo se forem considerados apenas os animais juvenis. No entanto, o comprimento do esôfago encontrado em um animal adulto por Magalhães et al. (2010) foi de $24,70 \mathrm{~cm}$, sendo o CCC e a LCC deste animal de $108,20 \mathrm{~cm}$ e $106,50 \mathrm{~cm}$, respectivamente. Esse dado apresenta-se muito discrepante quando comparado com o tamanho do esôfago do animal adulto avaliado no presente estudo, entretanto, não existem dados na literatura que justifiquem essa diferença no comprimento do órgão em animais adultos. Dessa forma, é de extrema necessidade a realização de novos estudos, a fim de se obterem mais parâmetros com o intuito de esclarecer essas divergências.

Em estudo realizado por Nakashima (2008), os exemplares analisados apresentaram medidas de comprimento curvilíneo da carapaça variando entre 26 e $67 \mathrm{~cm}$, sendo considerados todos juvenis, bem como uma dieta onívora, ainda que composta principalmente por matéria vegetal, consistindo basicamente de algas e matéria animal, indicando que as tartarugas-verdes analisadas estavam no período de mudança de hábitos alimentares, carnívoro para herbívoro.

De acordo com Bolten (2003), os indivíduos juvenis desenvolvem uma estratégia de alimentação oportunista, predando organismos planctônicos, muitas vezes agregada em zonas frontais. Estudo realizado por Pupo et al. (2006) confirmou que, com exceção da tartaruga-oliva $\mathrm{e}$ da tartaruga-de-couro, todas as outras que ocorrem na costa brasileira preferem águas rasas para alimentação. Isso torna muito comum a presença de tartarugas próximo à costa e as consequentes capturas incidentais.

Acredita-se que a ausência de dados em tartarugas-verdes adultas seja devido ao baixo índice de encalhe desses animais. Segundo Guebert (2008), os indivíduos juvenis aparecem em maior frequência e, por isso, estão sujeitos a taxas de mortalidade diferenciadas em relação aos adultos. Além disso, em estudo anterior, Guebert et al. (2007) afirmaram que os animais adultos estão presentes na área, contudo os registros de mortalidade são pouco frequentes, o que indica que talvez os adultos não sofram os mesmos impactos que os juvenis.

Pupo et al. (2006) observaram que as tartarugas capturadas incidentalmente pela pesca artesanal na Ilha de Santa Catarina apresentavam um CCC entre $52 \mathrm{~cm}$ e $58 \mathrm{~cm}$, o que indicou a ocorrência de tartarugas juvenis, sendo a maioria delas $C$. mydas e Caretta caretta. Dados semelhantes foram encontrados por Bahia e Bondioli (2010), em São Paulo, e por Awabid et al. (2013), no Rio de Janeiro.

Esses dados já foram descritos em C. mydas, por Magalhães et al. (2012),, e por Magalhães (2010) em Podocnemis sextuberculata e Podocnemis dumerilianus.

Pinheiro et al. (2010) descreveram as características do esôfago de Mesoclemmys vanderhaegei, que é contínuo caudalmente à cavidade oral. Apresenta-se como uma estrutura tubular, inicialmente localizada dorsalmente à traqueia (segmento cervical), desviando-se levemente para a direita à medida que se direciona caudalmente e, por fim, posicionandose à esquerda da traqueia e dorsalmente ao coração, ao entrar na cavidade celomática, imediatamente antes de desembocar no estômago, formando o segmento celomático.

Embora em tartarugas-verdes não existam relatos de utilização dos termos esôfago cervical e esôfago celomático, anatomicamente também é possível identificar essas duas porções.

Magalhães et al. (2012) obtiveram dados semelhantes ao estudarem a morfologia do tubo digestório de C. mydas. Entretanto, esses autores observaram uma região em forma de bolsa em dois animais, caracterizando um divertículo esofágico, o que não foi verificado neste estudo. Não se sabe, porém, se o divertículo aparece normalmente em alguns indivíduos ou se está relacionado a algum processo patológico prévio.

Em estudo realizado em Caretta caretta e Orlitia borneensis por meio de endoscopia, observaramse papilas ao longo do esôfago que se tornavam ausentes na região de transição para o esfíncter gastroesofágico, evidenciando uma mucosa pregueada (Pressler et al., 2003). Aspectos anatômicos distintos foram verificados no 
esôfago de Mesoclemmys vanderhaegei, em que a mucosa do segmento cervical é lisa e à medida que se direciona caudalmente, próximo ao estômago, apresenta pregas orientadas longitudinalmente (Pinheiro et al., 2010).

Valente et al. (2006) associaram as papilas esofágicas macroscopicamente às papilas encontradas na mucosa bucal de bovinos e ovinos. Segundo Lutz e Musick (1997), a contração do esôfago tem função de expelir o excesso de água pela cavidade oral e pelas narinas, e essas papilas a de reter o alimento sólido ingerido, processo durante o qual o esfíncter gastroesofágico se contrai, evitando a passagem de conteúdo para o estômago.

Por outro lado, Macedo et al. (2011) afirmaram que a presença das papilas queratinizadas e voltadas para a parte interna do aparelho digestório é um agravante anatômico em relação à ingestão de resíduos sólidos pelas tartarugas marinhas, uma vez que, como adaptação à alimentação no ambiente aquático, impedem o refluxo, fazendo com que resíduos seja mantidos.

A ausência de muscular da mucosa e submucosa no esôfago de $C$. mydas foi verificada por Magalhães et al. (2010). No entanto, Elliott (2007) citou que realmente há ausência da muscular da mucosa do esôfago em muitas espécies de répteis, mas esta pode ser encontrada em algumas espécies de tartarugas, e Sikiwat et al. (2013) descreveram, no esôfago de C. mydas juvenil, a presença de uma submucosa de tecido conjuntivo frouxo circundado por musculatura lisa, disposta circularmente, para permitir a passagem do alimento.

A existência de tecido mixomatoso no interior das papilas cônicas, chamadas de "espinhos esofágicos", foi verificada por Dunlap (1955), citado por Vogt et al. (1998), em estudo com tartarugas gigantes (Dermochelys coriacea). Em C. mydas não existem citações desse componente.

Vale ressaltar que o tecido mixoide é rico em ácido hialurônico e, portanto, seria melhor evidenciado por técnicas histoquímicas, como o Alcian Blue, que cora mucinas ácidas. Entretanto, neste estudo, utilizou-se apenas o PAS, que cora positivamente mucinas neutras; mesmo assim, foi possível identificar o tecido mixoide no interior das papilas. Acredita-se que esse tecido facilite a movimentação destas na porção anterior do esôfago.

Alguns aspectos diferentes dos encontrados neste estudo foram descritos por Magalhães et al. (2010) em C. mydas, os quais afirmaram que histologicamente o esôfago apresenta mucosa pregueada, com lâmina própria aglandular, formada por tecido conjuntivo frouxo, e afirmaram que a ausência de glândulas indica que esse órgão apresenta apenas função mecânica. Os autores descreveram também que a camada muscular é formada por musculatura estriada organizada em feixes em uma única camada, dispostos longitudinalmente, contendo abundante tecido conjuntivo frouxo entre os feixes. Acredita-se que a ausência da descrição do exato local de coleta das amostras esteja diretamente relacionada às diferenças histológicas observadas.

É possível afirmar, com os dados do presente estudo, que o esôfago de $C$. mydas possui função mecânica devido à queratinização do epitélio e do tecido mixoide existente na lâmina própria que sustenta as papilas cônicas, além da produção de muco pelas glândulas esofágicas da lâmina própria da mucosa na junção gastroesofágica, confirmada pela reação positiva ao PAS, as quais certamente auxiliam na propagação do alimento para o estômago.

Esses achados foram confirmados por Junqueira e Carneiro (2013), os quais citaram que a secreção produzida pelas glândulas esofágicas tem por função facilitar o transporte do alimento e proteger a mucosa. Entretanto, esses autores observaram as glândulas mucosas do esôfago na submucosa de outras espécies.

A presença das glândulas esofágicas em $C$. mydas também foi descrita por Santoro et al. (2007), que verificaram um parasito trematoda no interior das glândulas causando esofagite e adenite. Entretanto, os autores afirmaram que as lesões são frequentes na região distal do esôfago e demonstraram a presença de placas brancacentas em uma porção do órgão contendo pregas longitudinais sem papilas cônicas. É possível que a região citada pelos autores como distal seja a mesma que, neste estudo, denominou-se junção gastroesofágica, uma vez que não há papilas cônicas, e sim pregas 
reticulares longitudinais. tais achados também permitiram confirmar que esses animais possuem estruturas glandulares no esôfago.

Em outro estudo com as características do esôfago de Phrynops geoffroanus, verificaram-se também algumas diferenças histológicas, sendo as principais: a presença de glândulas bem definidas no epitélio e lâmina própria bem vascularizada na base do epitélio (Vieira-Lopes et al., 2014). Nesse mesmo contexto, Magalhães (2010) também evidenciou muitos aspectos distintos em cágados, como a mucosa revestida por dois tipos distintos de epitélio, epitélio estratificado pavimentoso e epitélio estratificado cilíndrico. Também foi verificado que, no epitélio estratificado cilíndrico, há presença de células superficiais mucosas com reação positiva ao PAS e não foi evidenciada a muscular da mucosa. A submucosa é aglandular, e a camada muscular externa é disposta em feixes de músculo intercalados com feixes de conjuntivo frouxo, composta de músculo liso.

Acredita-se que os hábitos alimentares dos cágados possam estar diretamente relacionados às características histológicas do esôfago, como o hábito de ingerir organismos vivos, pois, de uma maneira geral, as espécies de cágados podem ser consideradas onicarnívoras. Dessa forma, é possível sugerir que as diferenças histológicas nesse órgão são decorrentes dos hábitos alimentares dos animais.

\section{CONCLUSÃO}

O esôfago de C. mydas é um órgão tubular muscular constituído de papilas cônicas que variam de quantidade e tamanho à medida que se aproximam do estômago, revestidas por epitélio estratificado pavimentoso queratinizado e ricas em tecido mixoide. Há variação nas camadas mucosa, muscular externa e serosa e há presença de glândulas produtoras de muco na mucosa da região da junção gastroesofágica. As características histomorfológicas do esôfago de C. mydas são importantes para exercer a função mecânica e de proteção da mucosa desse órgão.

\section{AGRADECIMENTOS}

À empresa CTA - Meio Ambiente, por permitir a coleta de material; à Coordenação de Aperfeiçoamento de Pessoal de Nível Superior (Capes), pela concessão de bolsa de mestrado.

\section{REFERÊNCIAS}

ALMEIDA, A.P.; SANTOS, A.J.B.; THOMÉ, J.C.A. et al. Avaliação do estado de conservação da tartaruga marinha Chelonia mydas (Linnaeus, 1758) no Brasil. BioBrasil, v.1, p.18-25, 2011.

AWABDI, D.R.; SICILIANO, S.; BENEDITO, A.P.M.D. Ingestão de resíduos sólidos por tartarugasverdes juvenis, Chelonia Mydas (L. 1758), na costa leste do estado do Rio de Janeiro, Brasil. Biotemas, v.26, p.197-200, 2013.

BAHIA, N.C.F.; BONDIOLI, A.C.V. Interação das tartarugas marinhas com a pesca artesanal de cercofixo em Cananéia, litoral sul de São Paulo. Biotemas, v.23, p.203-213, 2010.

BJORNDAL, K.A. Foraging ecology and nutrition of sea turtles. In: LUTZ, P.L.; MUSICK, J.A. (Eds). The biology of sea turtles. Florida: CRC Press, 1997. p.199-231.

BOLTEN, A.B. Variation in sea turtle life history patterns: neritic vs. oceanic developmental stages. In: LUTZ, P.L.; MUSICK, J.A. The biology of sea turtle. 2.ed. Florida: Crc Press, 2003. p.243-258.

CASALE, P. Sea Turtle by-catch in the mediterranean. Fish Fish, v.12, p.299-316, 2011.

COSTA, M.F.; IVAR DO SUL, J.A.; SILVACAVALCANTI, J.S. et al. On the importance of size of plastic fragments and pellets on the strandline: a snapshot of a brazilian beach. Environ. Monit. Assess., v.168, p.299-304, 2009.

ELLIOTT, J.R. Overview of reptile biology, anatomy, and histology. In: Infectious diseases and pathology of reptiles. New York: Taylor \& Francis Group, 2007. p.1-25.

GUEBERT, F. M. Ecologia alimentar e consumo de material inorgânico por tartarugas verdes, chelonia mydas, no litoral do estado do Paraná. 2008. 76f. Dissertação (Mestrado Em Ciências BiológicasZoologia) - Universidade Federal do Paraná, Curitiba, PR.

GUEBERT, F.M.; ROSA, L.; LÓPEZ, E.A.B. et al. Monitoramento das tartarugas marinhas no litoral do estado do Paraná, sul do Brasil. In: JORNADA DE CONSERVACIÓN E INVESTIGACIÓN DE TORTUGAS MARINAS EN EL ATLÂNTICO SUR OCCIDENTAL, 3., 2007, Piriápolis, Uruguai. Anais... Piriápolis: ASO, 2007. p.79. (Resumo). 
JUNQUEIRA, L.C.; CARNEIRO, J. Histologia básica: texto e atlas. 10.ed. Rio de Janeiro: Guanabara Koogan, 2013. 540p.

LEWISON, R.L.; CROWDER, L.B.; READ, A.J. et al. Understanding impacts of fisheries bycatch on marine megafauna. Trends Ecol. Evol., v.19, p.598604. 2004.

LUTZ, P.L.; MUSICK, J.A. (Eds). The biology of sea turtles. Florida: CRC Press, 1997. 432p.

MACEDO, G.R.; PIRES, T.T.; ROSTÁN, G. et al. Ingestão de resíduos antropogênicos por tartarugas marinhas no litoral norte do estado da Bahia. Cienc. Rural., v.41, p.1938-1943, 2011.

MAGALHÃES, M.S. Morfologia do tubo digestório aplicada à compreensão da dieta em quelônios da família Podocnemididae. 2010. 78f. Dissertação (Mestrado) - Instituto Nacional de Pesquisas da Amazônia, Fundação Universidade do Amazonas, Manaus, AM.

MAGALHÃES, M.S.; FREITAS, M.L.; SILVA, N.B.; MOURA, C.E.B. Morfologia do tubo digestório da tartaruga verde (Chelonia mydas). Pesqui. Vet. Bras., v.30, p.676-684, 2010.

MAGALHÃES, M.S.; SANTOS, A.J.B.; SILVA, N.B.; MOURA, C.E.B. Anatomy of the digestive tube of sea turtles (Reptilia: Testudines). Rev. Bras. Zool., v.29, p.70-76, 2012.

MANGEL, J.C.; ALFARO-SHIGUETO, J.; WITT, M.J. et al. Post-capture movements of loggerhead turtles in the southeastern pacific ocean assessed by satellite tracking. Mar. Ecol. Prog. Ser., v.433, p.261272, 2011.

NAKASHIMA, S.B. Dieta da tartaruga-verde Chelonia mydas (Linnaeus, 1758) (Testudines, Cheloniidae) no litoral norte do Rio Grande do Sul. 2008. 38f. Dissertação (Mestrado - Programa de pósgraduação em biociências - Zoologia) - Faculdade de Biociências, Pontifícia Universidade Católica do Rio Grande do Sul, Porto Alegre, RS.

PINHEIRO, J.N.; GODOY, I.; BRITO, E.S. et al. Macroscopic aspects of the gastrointestinal tract of the south american freshwater turtle Mesoclemmys vanderhaegei (Bour, 1973). Braz. J. Vet. Res. Anim. Sci., v.47, p.429-438, 2010.
PRESSLER, B.M.; GOODMAN, R.A.; HARMS, C.A. et al. Endoscopic evaluation of the esophagus and stomach in three loggerhead sea turtles (Caretta caretta) and a malaysian giant turtle (Orlitia Borneensis). J. Zoo Wildl. Med., v.34, p.88-92, 2003.

PUPO, M. M.; SOTO, J.M.R.; HANAZAKI, N. Captura incidental de tartarugas marinhas na pesca artesanal da ilha de Santa Catarina, Sc. Biotemas, v.19, p.63-72, 2006.

REDLIST of threatened species, Version 2013.2. IUCN, 2013. Disponível Em: $<$ http://www.iucnredlist.org $>$ Acessado em: 2 Mai, 2014.

SANTORO, M.; MORALES, J.A.; STACY, B.; GREINER, E.C. Rameshwarotrema Uterocrescens Trematode parasitism of the oesophageal glands in Green Turtles. Vet. Rec., v.160, p.59-60, 2007.

SIKIWAT, S.; PUMIPAIBOON, M.; KAEWSANGIEM, S.; AREEKIJSEREE, $M$. Histological study of postmortem juvenile green sea turtle (Chelonia mydas) from royal thai navy sea turtle nursery, Phang-Nga, Thailand. Int. J. Biol., Vet., Agric. Food Eng., v.7, p.429-431, 2013.

VALENTE, A.L.S.; CUENCA, R.; ZAMORA, M.A.; et al. Sectional anatomic and magnetic resonance imaging features of coelomic structures of loggerhead sea turtles. Am. J. Vet. Res., v.67, p.1347-1353, 2006.

VIEIRA-LOPES, D.A.; NASCIMENTO, A.A.D.; SALES, A. et al. Histology and histochemistry of the digestive tract of Phrynops geoffroanus (Testudines, Chelidae). Acta Amaz., v.44, p.135-142, 2014.

VOGT, R.C.; SEVER, D.M.; MOREIRA, G. Esophageal papillae in pelomedusid turtles. $J$. Herpetol., v.32, p.279-282, 1998.

WALLACE, B.P.; LEWISON, R.L.; MCDONALD, S.L. et al. Global patterns of marine turtle bycatch. Conserv Lett.. v.3, p.131-142, 2010.

WYNEKEN, J. The anatomy of sea turtles. Miami: National Oceanic and Atmospheric Administration, 2001. 172p. (Noaa Technical Memorandum NmfsSefsc-470). 